\title{
Dynamic Bayesian Networks for Sequential Quality of Experience Modelling and Measurement
}

\author{
Karan Mitra ${ }^{\dagger \ddagger}$, Arkady Zaslavsky ${ }^{\dagger \ddagger}$, and Christer Åhlund $d^{\ddagger}$ \\ ${ }^{\dagger}$ Caulfield School of Information Technology, Monash University \\ 900 Dandenong Road, Caulfield East, Victoria, Australia, 3145 \\ ${ }^{\ddagger}$ Luleå University of Technology \\ SE-971 87 Luleå, Sweden \\ \{karan.mitra, arkady.zaslavsky, christer.ahlund\}@ltu.se \\ http://karanmitra.me/research
}

\begin{abstract}
This paper presents a novel context-aware methodology for modelling and measuring user-perceived quality of experience (QoE) over time. In particular, we create a context-aware model for QoE modelling and measurement using dynamic Bayesian networks (DBN) and a context-aware state-space approach. The proposed model is then used to infer and determine users' QoE in a sequential manner. We performed experimentation to validate the proposed model. The results prove that it can efficiently model, reason and measure QoE of the users'.
\end{abstract}

Keywords: Algorithm, Bayesian network, dynamic Bayesian network, context-awareness, quality of experience, quality of service

\section{Introduction}

Measuring user perceived quality of experience is a challenging task. This can be attributed to the fact that QoE not only depends on network parameters, but also on user's, device and the environmental context parameters $[24,13,3,15]$. These parameters together, affect users' expectations and their cognitive, behavioral and psychological states [24,22]. These states then dictate how they perceive the overall QoE. We argue that QoE of users' evolves over time i.e., based on previous interactions with the system, application or the service. By repeatedly using a service/object/tool, users' may get more accustomed and comfortable and measuring QoE at a single point in time and space will not yield correct measurements. Rather, their QoE needs to be assessed over a period of time (e.g., several days or several experimental runs) so that correct conclusions can be drawn.

Karapanos et al. [10] conducted a study concerning mobile phone usage. Their results show that users' perception of innovativeness inscreased during the first month and then remained stable. Also, users learnability was low for the first week and then increased sharply when users' got accustomed to their mobile 
devices. Perkis, Munkeby and Hillestad [19] conducted a 4 week study regarding QoE in 3G networks. Their results show that user expectations decreased after two weeks. They also show that the mean opinion score (MOS) regarding video application also decreased in the last two weeks from 3.4 to 3.1 which was measured on the scale of 1 to 5 . These results strongly suggests that users' QoE varies with time. In this paper, we focus on QoE related to multimedia applications such as voice over IP (VoIP). We note that the current state-of-the-art (see, $[14,24,21,15,3,2,5,23,9,11,4,17,19,22,12,13])$ does not address the problem of QoE measurement of multimedia applications over time. Mostly experiments are conducted in the lab settings or living lab settings [4] and then conclusions regarding QoE are drawn. This is particularly true for research done on voice and video quality assessment domain [16]. There is also no provision in the current ITU-T and ETSI recommendations and standards such as the ITU-T G.107 [5] and ETSI STF 354 [2] to measure QoE over time.

Thus, in this paper, we consider the problem of measuring QoE over time. We believe this will help stakeholders such as network and codec engineers and application designers [3] in: 1.) understanding customers behavior over time and hence, helping them in correctly measuring customers QoE; 2.) providing customized and personalized services to customers in the form of user-centric SLAs and value-added services and applications; and 3.) creating opportunities for network/services/codec optimizations based on the feedback provided by the users in realistic settings, and by correctly inferring the root causes associated with their feedback. Unlike the state-of-the-art, we formulate QoE assessment problem as a sequential reasoning and inference problem where after each test, users' assess the QoE offered by the network operators by consciously or subconsciously bearing in mind the previous interactions.

We apply context spaces theory [18] to model users' QoE and dynamic Bayesian networks (DBN) [20] to determine the overall QoE of users'. Recently, Mitra et. al $[12,13]$ developed a decision-theoretic approach for QoE modelling and measurement in mobile and pervasive computing environments. In this paper, we extend their approach to incorporate sequential QoE measurement. In all, we present the following contributions: 1.) we present a novel approach for QoE estimation as a context-aware sequential decision making problem which is not considered by practitioner's till-date. In particular, we formulate this problem as an inference problem in the light of QoE estimation and 2.) we extend a general context model [18] to include sequential data fusion and inference.

In section 2, we present the related work. In section 3, we present our methodology to measure QoE. In section 4, we present the results and a discussion for the same. Finally, section 5 presents the conclusion and future work.

\section{Related Work}

Sung [22], developed algorithms and architectures for QoE reasoning and adaptation. However, their approach was limited to mobile video applications. Wu et $a l$. [24] define and classify QoE related parameters and try to find the correlation 
between the QoE and QoS related classes. Their approach can be impractical when there are several QoE and QoS parameters as finding correlations between each and every parameter is a complex task. Furthermore, they do not consider the use of user and device related context parameters. Moller et al. [15], provides a taxonomy based on which application designers can select relevant QoE and QoS parameters to build multimedia applications. However, they do not present methods for modelling QoS and QoE relationships. Brooks and Hestnes [3, 8] stress the need to consider a combination of subjective and objective methods to determine QoE. Mitra et al. [12,13], developed a context-aware, decisiontheoretic approach for QoE measurement using Bayesian networks and utility theory. Their results show that they can achieve $94 \%$ accuracy for QoE estimation for two QoE classes. Karapanos et al. [10] suggests the need for finding causal relations between QoE parameters over time.

We gather that none of the techniques mentioned in the state-of-the-art address the problem of sequential QoE assessment. We state that a unified framework for sequential QoE modelling and measurement is required. Thus, in this paper, we develop and present a novel context-aware methodology for QoE modelling, reasoning and inference over time.

\section{Context-Aware Quality of Experience Modelling and Measurement Using State-Space Approach and Dynamic Bayesian Networks}

In this section, we present a methodology for sequential modelling, reasoning and measurement of QoE experienced by users'. Our model uses the cues collected from context sources such as sensors and network probes as observations and measures the QoE over time. We consider context spaces theory (CST) [18] for modelling context information and then extend it to DBNs to measure QoE. The main premise behind CST is the mapping of context information/attributes onto context states. These context states are fused to determine the overall situation occurring at a particular point in time. Formally, there are three important concepts in CST: context attributes defined as $a_{i}^{t} \in A$; context states defined as $S_{i}^{t} \in S$; and situation spaces defined as $R_{i}^{t} \in R$.

A context attribute, $a_{i}^{t}$, is the data that is used to infer a situation. For example, GPS coordinates $\left(a_{G P S}^{t}\right)$, delay $\left(a_{\text {delay }}^{t}\right)$, jitter $\left(a_{\text {jitter }}^{t}\right)$ and packet loss $\left(a_{P L}^{t}\right)$. A context state is the current state of the user or application at time $t$. It contains a number of context attributes to represent the state. A particular context state comprising of several context attributes is represented as $S_{i}^{t}=$

$\left(a_{i}^{t}, \ldots, a_{n}^{t}\right)$. For example, consider a VoIP application with $a_{\text {delay }}^{t}=20 \mathrm{~ms}$ and $a_{P L}^{t}=0 \%$ packet loss. The resultant MOS calculated using the ITU-T E-Model [5] would be around 4 . Thus, the context state, user satisfaction $\left(S_{U S}^{t}\right)$ is "very good" by assuming $S_{U S}^{t}$ is measured on a 1 to 5 Likert scale. Where 1 represents "poor", 5 represents "excellent" and 2 and 3 represents "fair" and "good", respectively. If the application is used by the user at his/her home, another context attribute, location $\left(a_{l o c}^{t}\right)$, is added to infer the same context state. Finally, 


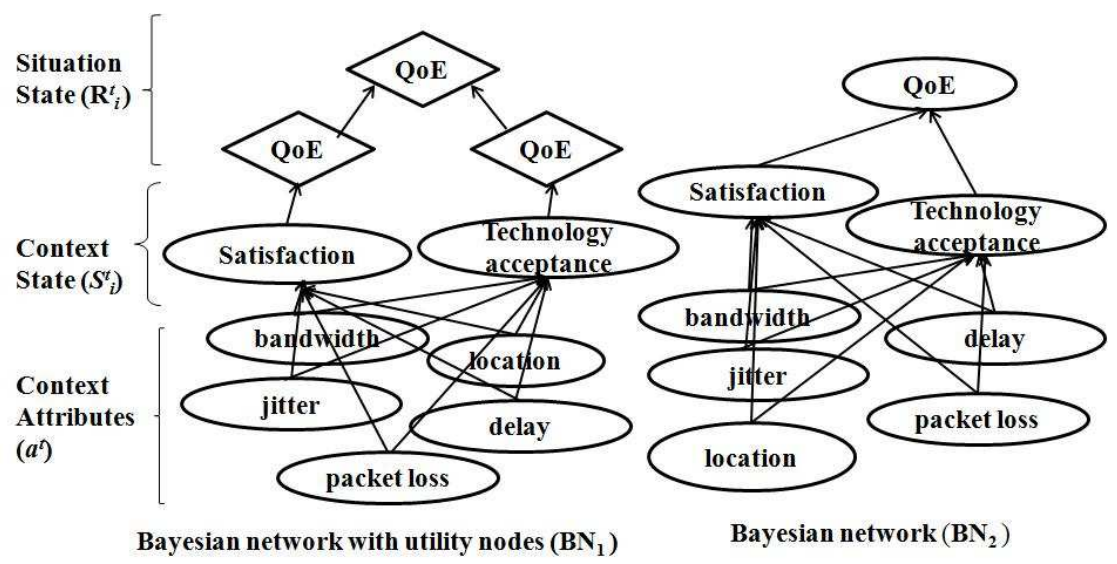

Fig. 1. BN with utility nodes $\left(B N_{1}\right)$ converted to a simple $\mathrm{BN}\left(B N_{2}\right)$ to incorporate a new chance node, QoE. All the utility nodes are converted back to chance nodes by performing linear scale transformation and re-learning the model parameters.

a situation space represents a real-life situation. It is a collection of regions of attribute values corresponding to a predefined situation. It is denoted by a vector space $R_{i}=\left(a_{1}^{R}, \ldots, a_{n}^{R}\right)$ where an acceptable region, $a_{n}^{R}$, is defined by the set of variables $V$ which satisfies the predicate $P$ i.e., $a_{n}^{R}=\{V \mid P(V)\}$. Considering the aforementioned context states, the overall situation would be "user is in his home and his QoE is very good".

Before defining a DBN for QoE measurement, we need to define a simple context-aware QoE model based on static BN and utility nodes. We refer to notations of [20] to define a BN as a directed acyclic graph (DAG) where random variables form the nodes of a network. The directed links between the nodes form the causal relationships. The direction of a link from $\mathrm{X}$ to $\mathrm{Y}$ means that $\mathrm{X}$ is the parent of Y. Any entry in the network can be calculated using the joint probability distribution (JPD) denoted as:

$$
P\left(x_{i}, \ldots, x_{n}\right)=\prod_{i=1}^{n} P\left(x_{i} \mid \text { Parents }\left(X_{i}\right)\right)
$$

Where, Parents $\left(X_{i}\right)$ is the parent of $x_{i}$. Figure 1 shows our BN which is extended to include utility nodes to calculate the overall QoE. At the lowest level, context information such as bandwidth $\left(a_{\text {bandwidth }}^{t}\right)$ and location $\left(a_{\text {location }}^{t}\right)$ is collected from sensors and network probes. At the intermediate level, context states such as technology acceptance $\left(S_{T A}^{t}\right)$ and user satisfaction $\left(S_{U S}^{t}\right)$ are inferred. These states are hidden. Finally, the top-most state is the situation state or the goal state which is inferred to determine the overall QoE situation of the user $\left(R_{Q \circ E}^{t}\right)$.

Based on [12], to infer $\left(R_{Q_{o E}}^{t}\right)$, expected utility of each context state is calculated. Using multi-attribute utility theory, these utilities are added together 


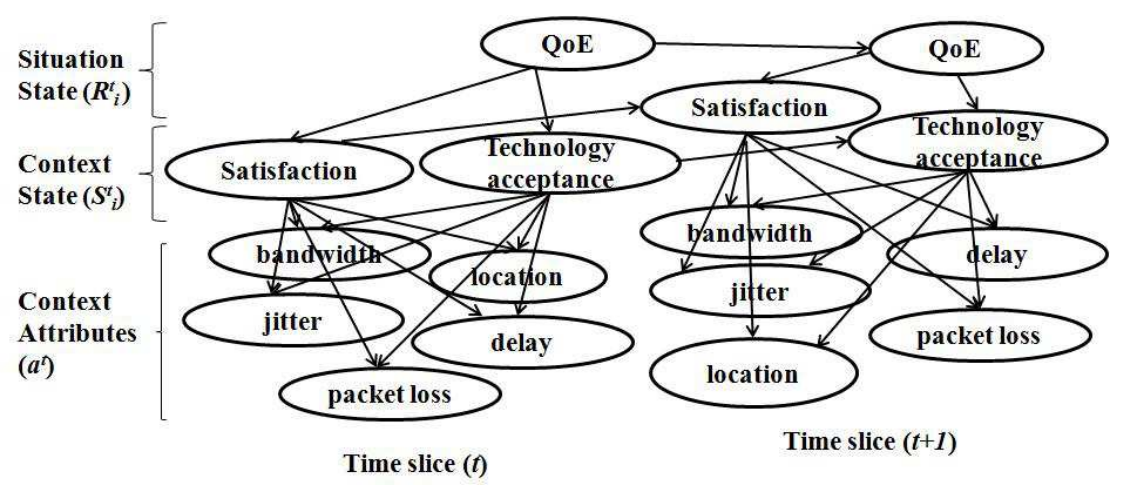

Fig. 2. DBN for QoE measurement and prediction. Two-time slices are assumed.

as a weighted sum to determine a scalar value. This scalar values in then linearly transformed on the scale of 0 and 1 and is mapped on the bi-polar scale to determined the QoE situation, $R_{Q o E}^{t}$. Once it is determined, a new $\mathrm{BN}\left(B N_{2}\right)$ is created and it is added to the $B N_{2}$ as a chance node and the network is re-learnt using the previous context attributes, states and the new QoE values.

\subsection{Sequential QoE Assessment and Measurement Using Probabilistic Inference}

In the previous section, we use CST, BN and utility theory for QoE modelling, assessment and measurement. However, the proposed approach did not consider QoE evolving over time. Thus, in this section, we develop a new probabilistic model for assessing and measuring QoE over time. This results in capturing the behaviour and experiences of a user that also evolves over time and which cannot be measured at only one instance and within a limited scenarios. User interacts with their system on a daily basis and generates new ratings. The resulting data is used to improve the model with better modelling and prediction capabilities. Once a $\mathrm{BN}$ is created, as mentioned in the previous section, we can unroll it further to formulate a DBN which can incorporate temporal inter-dependencies [20].

In our case, the strategy is to find an approximate hypothesis $\left(h_{n}\right)$ that best describes the QoE of users' at each experimental trail as we are dealing with partially observable environment and the user choices about QoE classes are not deterministic. To infer approximate QoE, we compute the marginals at each time step for the state sequences related to hidden variables such as $S_{T A}^{t}$, $S_{U S}^{t}$ and $R_{Q o E}^{t}$. In order to use the DBN, we unroll the $B N_{2}$ to several time slices as required by the stakeholders. This will include setting up of temporal dependencies (transition probabilities). In our case, we will use the data to learn the transition probabilities along with other parameters of the network. Figure 2 shows a simple DBN for QoE measurement over time (assuming two time slices). 
As can be seen in the figure, $B N_{2}$ is unrolled and is repeated along two time slices.

\subsection{Model Description and a Context-Aware Algorithm for QoE Assessment Over time}

In this paper, for our DBN, we assume a first order Markov process, i.e., current state depends only on the previous state. For example, $Q o E_{t+1}, S_{U S}^{t+1}$ and $S_{T A}^{t+1}$ are dependent only on previous states, i.e., $Q o E_{t}, S_{U S}^{t}$ and $S_{T A}^{t}$. Based on [20], We formally write it as a transition model defined as: $\mathrm{P}\left(S_{t} \mid S_{t-1}\right)$ where $S_{t}$ is the current state of the system. In our case, we have three different hidden context state variables: $S_{T A}^{t}, S_{U S}^{t}$ and $R_{Q o E}^{t}$ which are inferred by the observations or the context attributes $\left(A_{t}\right)$ such as bandwidth $\left(a_{\text {bandwidth }}^{t}\right)$ and location $\left(a_{\text {location }}^{t}\right)$. As shown in figure 2, the current observations or attributes are dependent on the current state variables and can be written as: $P\left(A_{t} \mid S_{t}\right)$. This is called as the sensor model. Additionally, prior or the initial probabilities of the states are defined. This is written as: $S_{U S}^{0}, S_{U S}^{0}$ and $Q o E_{U S}^{0}$. Using transition and sensor models and prior distribution, the complete joint distribution for the DBN can be written as:

$$
P\left(S_{0}, \ldots, S_{t}, A_{1}, \ldots, A_{t}\right)=P\left(S_{0}\right) \prod_{i=1}^{t} P\left(S_{i} \mid S_{i-1}\right) P\left(A_{i} \mid S_{i}\right)
$$

Using e.q.2, any query regarding QoE states and the context attributes can be answered. QoE measurement can be done in each state based on the inferring the probability of the hypothesis, $h_{n} \in S_{t}$. In particular, given the evidence or the context attributes $\left(A_{t}\right)$, we calculate the marginals $P\left(S_{t} \mid A_{t}\right)$ for all the context and situation states. For inference in our DBN, exact and approximate algorithms can be used based on the space and time requirements [20]. In this paper we use an exact algorithm called Clustering [20] to infer all the hidden states.

In addition to inferencing, we must perform learning based on the data collected from the test subjects. In DBNs, the problem of learning can be divided into two parts, i.e., learning the sensor model parameters and learning transition probabilities (transition model) between the state variables. We consider the use of expectation maximization (EM) algorithm [20]. In order to learn the model, data can be collected from the users' then parameters can be learnt. In EM algorithm, there are two main steps: E-step which compute posteriors over the states and M-step which adjusts the model parameters maximize the likelihood of posteriors calculated in the E-step. The algorithm to calculate $R_{Q o E}^{t}$ is given below. As we have the transformed $B N_{2}$ into a DBN, this algorithm calculates the QoE at each time step based on current and and previous states along with the context attributes. As mentioned previously, we assume that our model follows the $1^{\text {st }}$ order Markov process where only the current and only previous states are considered. It might happen that the state space is large or the $R_{Q o E}^{t}$ needs to be measured in an online recursive manner. We use a sliding 
window $(\mathrm{W})$ of a particular size to keep sufficient statistics for the model. For example, we can keep in history the states of past ten tests to infer the current QoE. After which, the latest observations and the states are added to the sliding window and the model is re-adjusted. This algorithm provide enough power to accurately determine QoE at each time interval of infinite size while remaining within the Markov framework.

A context-aware algorithm to learn and measure QoE sequentially.

1. Initialize the model and the sliding window (W).

(a) Using the collected data learn network parameters.

2. Project to the next time-step.

(a) Generate new observations.

(b) Based on the new observations and prior probabilities, infer hidden parameters (QoE states).

(c) Record the observations and the states. i. Append it to latest observations in the sliding window.

3. Re-learn the network parameters from the new observations.

4. Go to Step 1 and repeat until final state.

5. Select the states with arg max (QoE) using the most likely state algorithm.

Once the new observations are generated at experimental trial, our model infers all QoE states for the current time-step by keeping in memory previous QoE states. The inferred values are then appended to the observation set and the $\mathrm{W}$ is re-adjusted. Then EM is run again to re-estimate the model parameters. This process repeats till the final state is reached. After parameter estimation, we have the smoothed estimates of the QoE for each time slice. To determine the most likely states, we use the most likely state algorithm [20]. This algorithm performs filtering on the all the states to find the most like state in linear-time.

\section{Results Evaluation}

\subsection{Experimental setup}

For results evaluation, we considered QoE assessment related to VoIP application that can use both ITU-T G.729 narrow band (NB) and ITU-T G.722 wide band (WB) codecs. We considered two QoE metrics, user satisfaction (US) and technology acceptance (TA) represented as state variables, $S_{T A}^{t}$ and $S_{U S}^{t}$ to measure the overall QoE $\left(R_{Q o E}^{t}\right)$. In this paper, US is represented as the mean opinion score (MOS) and is calculated on the scale of 1 to 5 . Where, 1 means "poor" and 5 means "excellent". "fair", "good" and "very good" are represented as 2, 3 and 4, respectively. TA is classified as a behavioural factor [13] that determines whether the user will use the technology, application or the service again by consciously or sub-consciously observing the underlying QoS and environmental related context. TA is calculated as a boolean variable represented as either "yes" or "no" to represent "user will accept the technology" and "user will reject the technology", respectively. 
Table 1. Selected context attributes and ranges.

\begin{tabular}{|c|c|}
\hline context attributes $\left(a_{n}\right)$ & values (ranges) \\
\hline \hline codec & ITU-T G.729, ITU-T G.722 \\
\hline packet loss $(\%)$ & low $(0 \%-5 \%)$, medium $(5 \%-7 \%)$, high $(7 \%-10 \%)$ \\
\hline delay $(\mathrm{ms})$ & low $(0 \mathrm{~ms}-150 \mathrm{~ms})$, medium $(150 \mathrm{~ms}-300 \mathrm{~ms})$, high $(>300 \mathrm{~ms})$ \\
\hline location & home, tram stop, office \\
\hline
\end{tabular}

Experimental scenarios and the choice of data set: We generated data simulating five users to be assessed over a period of six weeks. The choice of the number of users' is selected due to fact that in the living-labs settings such as [4], it is very difficult and expensive to collect user data over a period of several days and usually studies are conducted with a limited number of test subjects. We would also like to determine whether a small data set can be used to obtain satisfactory results. In our simulations, we considered a case where users' randomly give ratings based on the VoIP calls made at various times of the day. In all, we expect users' to make at least four calls daily (per user). Thus, every day, we expect 20 observations for all the test subjects. We used a non-overlapping time-window (W) of 1 week i.e., the overall QoE is predicted at the end of one week using 140 observations taken together. Indeed, stakeholders can adjust the size of $\mathrm{W}$ according to their needs. Based on $\mathrm{W}$, we train the DBN for all the observations generated at every week for six weeks.

Based on these observations, we generated data for six weeks related to user satisfaction (US) and technology acceptance (TA) for both the codecs based on ITU-T recommendations $[5,6]$. Table 1 shows the values and ranges for several context parameters. We set TA according to US i.e., if US lies between 3-5, the user will most likely accept the technology, else he/she will reject it. It was shown in [19], that user tends to give a lower score at the end of four week period. Thus, we bias the calculated US using this intuition and reduce the US by 2 (from "excellent" to "good"), uniformly, over a period of four weeks. Based on this, TA is also reduced accordingly as shown in figure 3. We further manipulated the conditional probability tables (CPTs) to include the effects of location on the overall QoE based on the fact that users' social context changes at different locations [8]. For example, we expect a user to be more comfortable at home rather than his/her office due to stress or background noise which might affect his/her QoE. This is reflected by assigning higher probability to home followed by tram stop and the office to maximize $R_{Q o E}^{t}$. Context states $S_{U S}^{t}$ and $S_{T A}^{t}$ are inferred and then fused together to determine $R_{Q o E}^{t}$.

\subsection{Results Analysis}

We developed a prototype using the GeNIe/SMILE package and APIs [1]. We used exact algorithm called Clustering [20] as the DBN inference algorithm. Figure 3 shows our DBN used for results evaluation. We consider finite horizon cases where we already know the finish time $\left(T_{f}=6\right)$ of the assessment period 
in advance (set by stakeholders). At each time-step $\left(t_{s}\right)$, QoE is inferred by our algorithm. Using the simulated data, parameters of the DBN are learnt using the expectation maximization algorithm (EM) [7,20], as shown in our algorithm. Indeed, data generated at each experimental run can be based on any number of test cases. As can be seen in figure 3, QoE of users' decreases uniformly from $t_{1}$ to $t_{3}$ and remains same at $t_{4}$ while using ITU-T G.729 codec, and the model is able to correctly track the varying QoE. This validation was performed using the leave-one out cross-validation procedure to test whether the proposed model is able to correctly estimate the QoE values over time. We can notice that the proposed model can correctly determine the most likely state sequence for QoE from time $t_{1}$ to $t_{4}$.

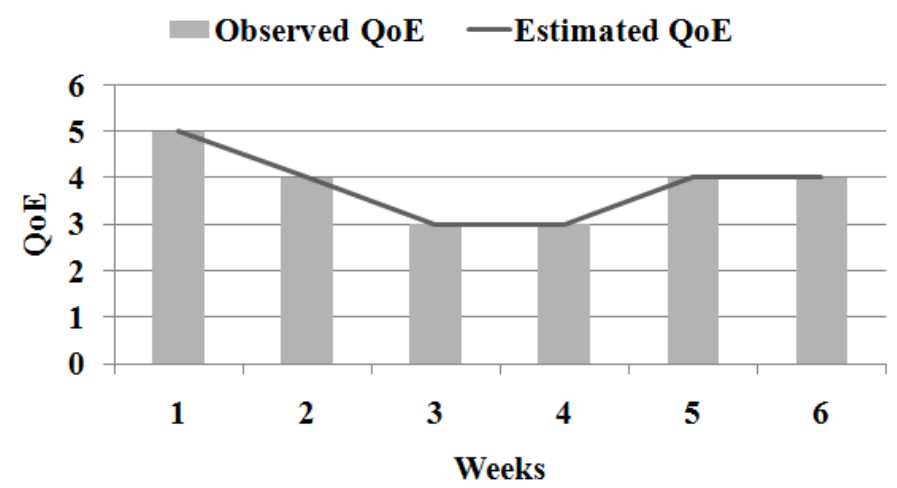

Fig. 3. Observed and estimated QoE values for six weeks. We can see that DBN is able to correctly match the observed QoE values.

To further validate the proposed model, we consider another case, where after assessing users' QoE over time, a smart VoIP application decides to change the codec proactively such that users' QoE is maximized. We simulate this case by changing the codec from NB to WB once the QoE of the user is predicted to fall below or remain constant for some time at a particular threshold ( 3 for MOS) at the end of four-week $\left(t_{4}\right)$. It was shown in [16], that ITU-T G.722 codec gives higher QoE than NB codecs such as ITU-T G.711 and ITU-T G.729 codecs. Thus, we generated data based on this observation, for time $t_{5}$ to $t_{6}$ and use our algorithm to re-estimate by re-learning the model parameters. As can be seen in figure 3, QoE of users' increases from 3 to 4 after the codec change at time $t_{5}$ and then remains constant at $t_{6}$. Figure 3 , shows the true and predicted estimates of the QoE values at each $t$. We can notice that the QoE decreases with time from week 1 to week 4 and it then starts to increase from week 5 due to the changes made by the smart application. We show that how our model is able to correctly match the true QoE estimates. We again performed leave-one-out cross-validation to test whether the proposed model is able to correctly estimate 
the $R_{Q o E}^{t}$ values. From these results, we can gather that the proposed model is able to establish causal relationships between several QoE states and is able track the variations in QoE involving over time.

\subsection{Discussion}

Figure 3, shows the true and predicted estimates of the QoE values at each $t$ (for six weeks). It shows that the QoE decreases with time from $t_{1}$ to $t_{4}$ and it then starts to increase from $t_{5}$ due to the codec changes made by the smart application. We show how our model is able to correctly match the true QoE estimates using leave-one-out cross-validation based on three QoE states $\left(S_{T A}^{t}, S_{U S}^{t}\right.$ and $\left.R_{Q o E}^{t}\right)$ and several context parameters $a_{P L}, a_{\text {delay }}, a_{\text {location }}$ and $a_{\text {codec }}$, over time. Our model is flexible as it allows the addition and deletion of QoE states and context attributes as and when required. It ensures that QoE states can be measured correctly under uncertainty using DBNs. This is achieved by fusing these states probabilistically based on current observations and prior estimates of the states. This helps to determine the overall QoE in a realistic settings based on the experiences of the users'. We are currently investigating the use of our QoE measurement model to incorporate several other QoE classes by involving more use case scenarios in our study to further validate the proposed model.

\section{Conclusion and Future Work}

In this paper, we present a novel context-aware methodology for quality of experience (QoE) modelling and measurement over time. The proposed methodology is based on a state space approach and dynamic Bayesian networks. It can incorporate several QoE classes and context parameters to accurately determine and predict QoE over time. We use the expectation maximization algorithm to deal with learning under uncertainty and missing user data. Our methodology is beneficial for network operators, codec engineers or application designers who are interested in measuring users' QoE in realistic settings. To the best of our knowledge, this is the first such attempt to measure QoE over time. In future, we will extend this methodology to incorporate sequential decision making in heterogeneous access networks.

\section{References}

1. Genie software package, http://genie.sis.pitt.edu/about.html, [online] access date: $02 / 01 / 11$.

2. ETSI STF 354. Guidelines and tutorials for improving the user experience of real-time communication services.

http : //portal.etsi.org/stfs/STF_HomePages/STF354/Default.aspx?Selection = Home 
3. P. Brooks and B. Hestnes. User measures of quality of experience: why being objective and quantitative is important. Network, IEEE, 24(2):8-13, march-april 2010.

4. K. De Moor, I. Ketyko, W. Joseph, T. Deryckere, L. De Marez, L. Martens, and G. Verleye. Proposed framework for evaluating quality of experience in a mobile, testbed-oriented living lab setting. Mob. Netw. Appl., 15:378-391, June 2010.

5. ITU-T Recommendation G.107. ITU-T Recommendation G.10\%, Methods for subjective determination of transmission quality, 2008.

6. ITU-T Recommendation G.113. Appendix I: Provisional planning values for the equipment impairment factor Ie, 1998.

7. D. Heckerman. A tutorial on learning with bayesian networks. In Proceedings of the NATO Advanced Study Institute on Learning in graphical models, pages 301-354, Norwell, MA, USA, 1998. Kluwer Academic Publishers.

8. B. Brooks, P. Hestnes and Heiestad. QoE (quality of experience) - measuring qoe for improving the usage of telecommunications services. Technical report, 2006.

9. S. Jumisko-Pyykkö and M. M. Hannuksela. Does context matter in quality evaluation of mobile television? In Proceedings of the 10th international conference on Human computer interaction with mobile devices and services, MobileHCI '08, pages 63-72, New York, NY, USA, 2008. ACM.

10. E. Karapanos, J. Zimmerman, J. Forlizzi, and J. Martens. Measuring the dynamics of remembered experience over time. Interacting with Computers, 22(5):328-335, 2010.

11. L. Liu, W. Zhou, and J. Song. The research of quality of experience evaluation method in pervasive computing environment. In Pervasive Computing and Applications, 2006 1st International Symposium on, pages 178-182, 2006.

12. K. Mitra, C. Åhlund, and A. Zaslavsky. A decision-theoretic approach for quality of experience measurement and prediction. In Proceedings of the 2011 International Conference on Multimedia and Expo (ICME), 2011.

13. K. Mitra, A. Zaslavsky, and C. Åhlund. A probabilistic context-aware approach for quality of experience measurement in pervasive systems. In Proceedings of the 2011 ACM Symposium on Applied Computing (SAC), 2011.

14. S. A. Moebs. A learner, is a learner, is a user, is a customer: Qos-based experienceaware adaptation. In $M M$ '08: Proceeding of the 16th ACM international conference on Multimedia, pages 1035-1038, New York, NY, USA, 2008. ACM.

15. S. Moller, K.-P. Engelbrecht, C. Kuhnel, I. Wechsung, and B. Weiss. A taxonomy of quality of service and quality of experience of multimodal human-machine interaction. In Quality of Multimedia Experience, 2009. QoMEx 2009. International Workshop on, pages 7-12, 29-31 2009.

16. S. Moller, M. Waltermann, B. Lewcio, N. Kirschnick, and P. Vidales. Speech quality while roaming in next generation networks. In Communications, 2009. ICC '09. IEEE International Conference on, pages 1-5, 2009.

17. Nokia. Quality of Experience (QoE) of mobile services: can it be measured and improved?, white paper, 2006.

18. A. Padovitz, S.W. Loke, A. Zaslavsky, B. Burg, and C. Bartolini. An approach to data fusion for context awareness. Modeling and Using Context, pages 353-367.

19. A. Perkis, S. Munkeby, and O.I. Hillestad. A model for measuring quality of experience. In Signal Processing Symposium, 2006. NORSIG 2006. Proceedings of the 7th Nordic, pages 198-201, 2006.

20. P. Norvig, and S. Russel. Artificial Intelligence: A modern approach. 2 edition, 2006. 
21. D. Soldani. Means and methods for collecting and analyzing qoe measurements in wireless networks. In World of Wireless, Mobile and Multimedia Networks, 2006. WoWMoM 2006. International Symposium on a, pages 531-535, 2006.

22. J. Sung. Football on Mobile Phones: Algorithms, Architectures and quality of experience in streaming video. $\mathrm{PhD}$ thesis, Umeå University, 2006.

23. W. Key and W. Greger. Determining utility functions for streaming low bitrate football video. In Ninth International Conference on Internet and Multimedia Systems and Applications, 2005.

24. W. Wu, A. Arefin, R. Rivas, K. Nahrstedt, R. Sheppard, and Z. Yang. Quality of experience in distributed interactive multimedia environments: toward a theoretical framework. In MM '09: Proceedings of the seventeen ACM international conference on Multimedia, pages 481-490, New York, NY, USA, 2009. ACM. 